\title{
CONQUISTANDO AMBIENTE SUBMARINO: SIMULAÇÃO 3D DE UM ROV COM APLICAÇÃO DE CONTROLE PROPORCIONAL DE POSE
}

\author{
Yuri Oliveira1; Matheus da Silva²; Leizer Schnitman³; Marco dos Reis ${ }^{4}$ \\ ${ }^{1}$ Universidade Federal da Bahia; Salvador/Bahia; yuri.matos@gmail.com; \\ 2Universidade Federal da Bahia; Salvador/Bahia; \\ 3Universidade Federal da Bahia; Salvador/Bahia; \\ ${ }^{4}$ Universidade Federal da Bahia - PPGM, SENAI CIMATEC; Salvador/Bahia;
}

Resumo: Este material faz uma comparação sobre duas estratégias de controle, o proporcional e manual, para o controle de posição e orientação do RexROV. A abordagem desta comparação é realizada através do UUV simulator, uma plataforma, que juntamente com o ROS e Gazebo, permite realizar simulações de ROV e AUV. Após a implementação das estratégias de controle, duas tarefas são executadas utilizando ambas as estratégias, e os resultados que são obtidos destas tarefas, formam a principal base para a análise de eficiência. É possível observar após esses testes que o controle proporcional possui um resultado melhor que o controle manual.

Palavras-Chave: RexROV; Controle proporcional; UUV.

\section{EXPLORING UNDERWATER ENVIRONMENT: 3D SIMULATION OF ROV WITH PROPORTIONAL POSE CONTROL}

\begin{abstract}
This paper compares proportional and manual pose control of RexROV underwater vehicle. The approach of this comparison happens with the use of UUV Simulator, a platform that, together with ROS and Gazebo, allows to perform ROV and AUV simulations. After the implementation of the control strategies, two tasks were performed, and its results, formed the main basis for the efficiency analysis. With the analysis of the results, which are properly demonstrated in this material, it is possible to observe that proportional control has a better result than manual control.
\end{abstract}

Keywords: RexROV, Proportional controller, UUV. 


\section{INTRODUÇÃO}

As explorações em ambiente submarino são de grande importância para a dinâmica da sociedade em aplicações industriais, científicas, militares [1]. A utilização de veículos, que conseguem se deslocar nesses espaços, aumenta a capacidade de exploração e possibilita a execução de uma grande variedade de operações. Em diferentes localidades, diversas empresas e centros de pesquisa estão concentrando interesse em explorações submarinas, em função deste crescente empenho, estudos sobre a implementação de ROVs e AUVs estão sendo realizados.

AUVs (Autonomous Underwater Vehicle), assim como o próprio nome já sugere, são veículos submarinhos que operam sem intervenção ou com uma baixa intervenção humana, no qual sistemas de navegação, direção e controle estão implementados. Assim como os AUVs, os ROVs (Remotely Operated underwater Vehicle) são também veículos submarinos, porém são normalmente controlados manualmente e consequentemente a eficiência das operações está fortemente conectada com a experiência do operador [2].

Tendo em vista o avanço e melhoria na navegação desses veículos submarinos, este trabalho teve objetivo de desenvolver um controle proporcional da posição e da orientação do veículo submarino fictício RexROV, de tal forma que o controle automático proposto fosse superior ao manual. Para realizar tal projeto, se utilizou um ambiente virtual que simula a dinâmica de um oceano [3], tal ambiente foi desenvolvido a partir do ROS [4] e Gazebo [5], o que possibilitou o teste do controle elaborado.

\section{METODOLOGIA}

O veículo utilizado no trabalho se locomove quando algum dos seus 8 propulsores é acionado. Seria complicado de controlar manualmente o RexROV somente pelo acionamento desses motores, dessa forma, para facilitar o controle humano do ROV, seu desenvolvedor implementou um controle de velocidade. Tal controle consiste em determinar as velocidades com que o veículo se deslocará em cada um dos seus 6 graus de liberdade, que consistem no deslocamento em $\mathrm{x}, \mathrm{y}$ e $\mathrm{z}$, e nas rotações em torno destes, que também são chamadas respectivamente de roll, pitch e yaw.

Normalmente a velocidade em cada eixo é estabelecida com o auxílio de um joystick que se comunica com a simulação no Gazebo através do ROS, mas para ter a liberdade de definir o valor exato desejado a qualquer momento, se desenvolveu um algoritmo em $\mathrm{C}++$ que substitui o dispositivo. Esse programa possibilitou $\mathrm{o}$ desenvolvimento de um sistema de controle proporcional de psoe, o qual tem como entrada os valores de pose desejados nos seus 6 graus de liberdade e tem como saída os valores de velocidades necessárias para se atingir a pose desejada.

Sendo assim, o problema de movimentação do RexROV ficou dividido como seis sistemas SISO (single input single output), sem considerar as perturbações que 
um movimento causa no outro. De forma a minimizar essas pertubações, a velocidade do veículo foi limitada.

Figura 1. Representação dos graus de liberdade do RexROV

Fonte: Autores, 2019

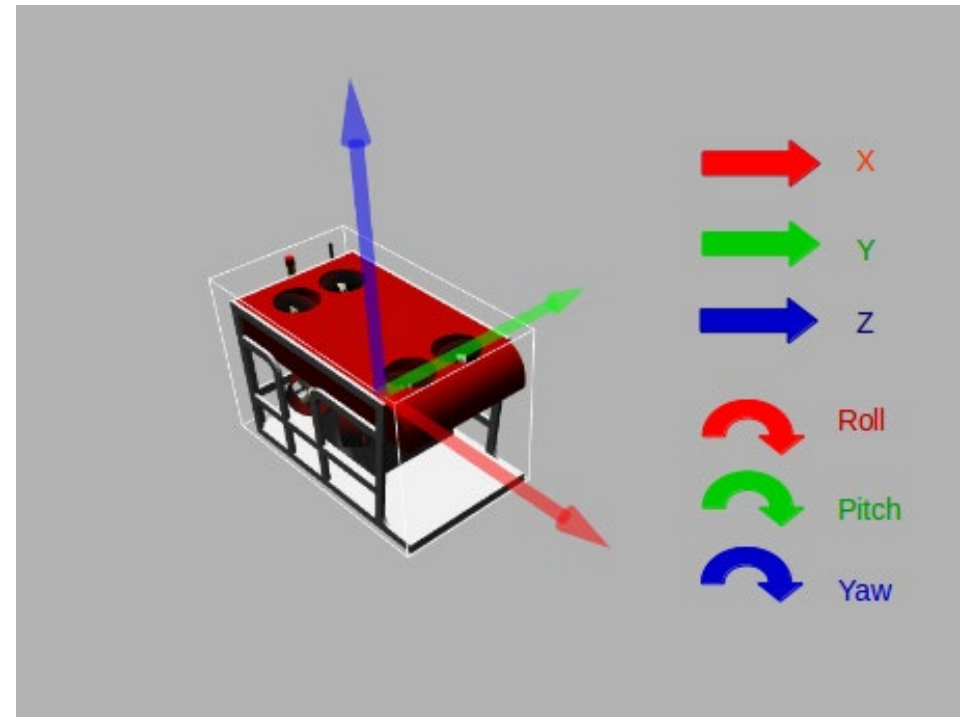

\subsection{Controle proporcional em cascata}

O controle de pose poderia ser desenvolvido para controlar a aceleração dos propulsores, porém não há necessidade desenvolver tal sistema, porque o controle de velocidade interno realiza justamente tal controle. Adicionalmente, esse sistema de controle está com um bom funcionamento e fornece as saídas de aceleração nos 6 DOF (Degrees of Freedom). O controle de velocidade interno funciona levando o ROV para a velocidade desejada, mesmo que ocorram interferências de correntes marítimas e falha nos motores. Considerando esses aspectos, a aplicação do controle da posição em cascata com o de velocidade é uma alternativa prática e eficaz de ser aplicada, sendo esses os motivos que levaram a estratégia ser escolhida.

A figura 2 abaixo representa o diagrama de controle do projeto. Primeiramente uma entrada de pose é fornecida, tal pose tem uma referência padrão, ou seja, sua posição e orientação estão em relação à um sistema de coordenadas fixo, que pode ser definido, por exemplo, por uma pose do ambiente em que ele vai atuar ou pela embarcação de lançamento. Por isso é necessário o conversor de coordenadas, para fazer com que a pose de entrada esteja diretamente relacionada ao sistema de coordenadas do RexROV, visto que a sua velocidade também está. 
Figura 2. Diagrama de Controle

Fonte: Autores, 2019

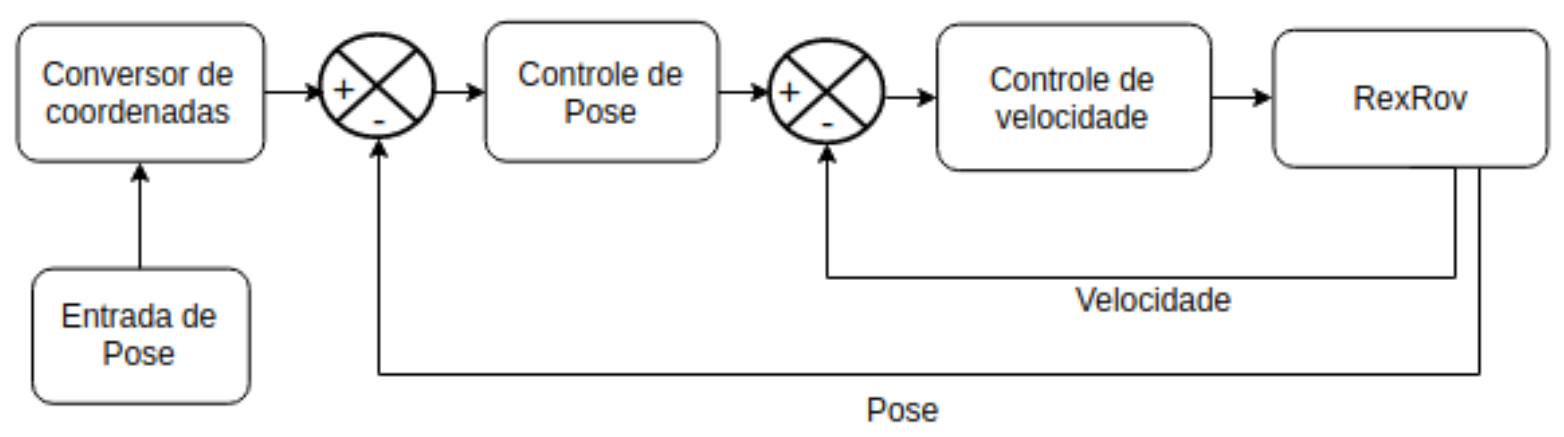

O controle em cascata consiste na associação de controladores, sendo o controlador escravo responsável por controlar uma variável interna, que nesse caso é a velocidade, e o controlador mestre responsável por controlar a variável desejada, que nesse caso é a pose [6]. Essa associação facilita o desenvolvimento do controlador de posição, pois esse atua na entrada do controlador de velocidade, ajustando essa variável para aproximar o ROV da posição desejada (figura 2) não sendo preciso atuar diretamente nos propulsores.

\subsection{Definição de constantes}

Os sistemas SISO, que estão sendo tratados pelo controlador de pose são integradores, visto que a posição é a integral da velocidade do objeto. Ao se fechar a malha de controle desse tipo de sistema, se obtém um sistema em malha fechada de primeira ordem e sua constante de tempo $\tau$ é igual ao inverso do ganho proporcional $K_{p}$. Desta forma, o valor de $K_{p}$ é inversamente proporcional a velocidade que o sistema atinge o setpoint. Porém, o sistema não é ideal e ocorre uma saturação na saída do controle, o que torna esse sistema instável ou oscilatório para valores elevados de $K_{p}$.

Com o objetivo de definir ganho adequado para o sistema, foi realizada uma análise de sensibilidade através da variação de valores de $K_{p}$. Com base nos resultados apresentados, observou-se que o sistema apresentava comportamento oscilatório nos DOF lineares para $K_{p} \geq 1,2$ e $K_{p} \geq 2,4 / \pi$ nos DOF angulares.

\section{RESULTADOS E DISCUSSÃO}

A teoria descrita acima foi desenvolvida e implementada no ambiente virtual de simulação subaquática UUV [3], dessa forma foi possível realizar testes e observar a eficácia do sistema em relação ao controle manual. Esse controle no RexROV é executado através de uma tele operação, na qual um operador usa um joystick que possibilita atuar em 4 graus de liberdade, sendo esses $x, y, z$ e yaw. Com o domínio destes 4 graus de liberdade, um operador, com um devido treinamento, pode alocar $o$ 
RexROV em qualquer posição no ambiente submarino, respeitando as limitações geométrica que este último impõe. Considerando que o objetivo do trabalho é de comparar o controle manual com o automático, somente esses 4 DOF foram observados.

Duas situações foram simuladas para comparar o desempenho das estratégias controle. A primeira teve como objetivo realizar um deslocamento sobre a posição do RexROV, partindo da coordenada $(30,40,-50)$ para $(31,41,-51)$, e sobre a orientação de partindo de $(0,0,0)$ para $(0,0,1)$, (roll, pitch, yaw). O segundo teste teve propósito avaliar o desempenho dos controladores diante de perturbações no sistema.

As Figuras 3 e 4, retratam as respostas nos 4 DOF especificados. Por meio da observação dos gráficos, é possível concluir que o controle automático é mais eficaz do que o manual, conseguindo executar os mesmos objetivos até 4 vezes mais rápido. Além disso, é perceptível que o controle manual, no segundo teste, não obteve êxito em se manter no setpoint na presença de perturbações, ao contrário do controle automático, que obteve sucesso em manter o ROV na pose desejada.

Figura 3. Rastreiamento de setpoint

Fonte: Autores, 2019

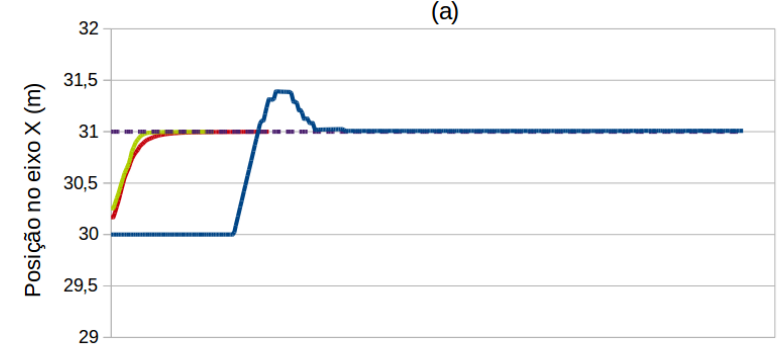

(c)

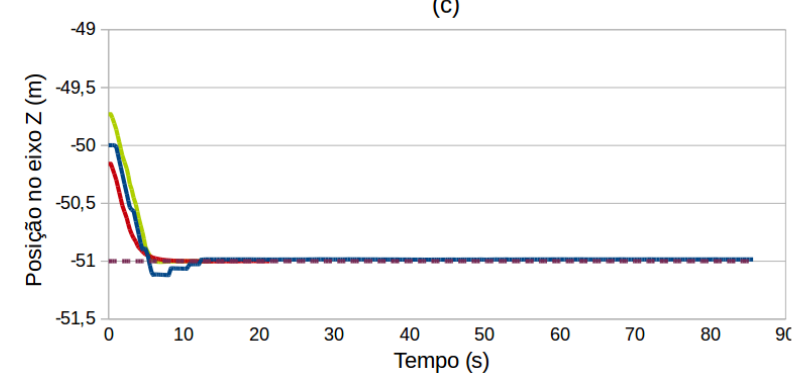

manual

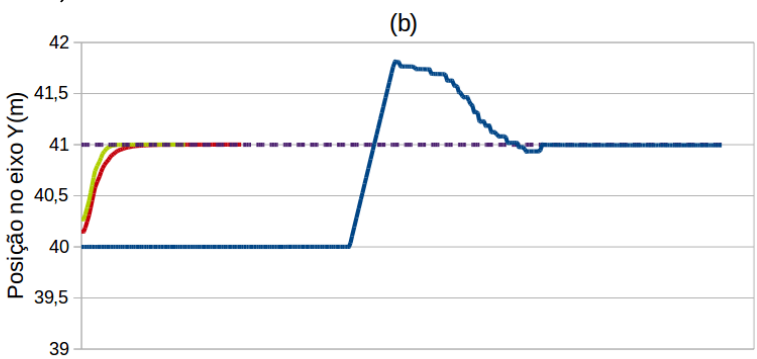

(d)

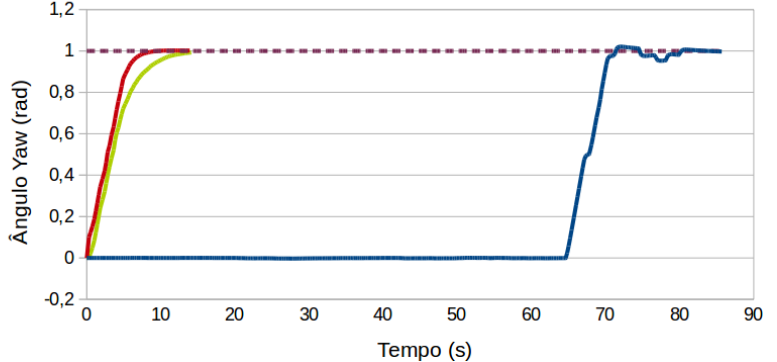


Figura 4. Rejeição de distúrbios

Fonte: Autores, 2019

(a)

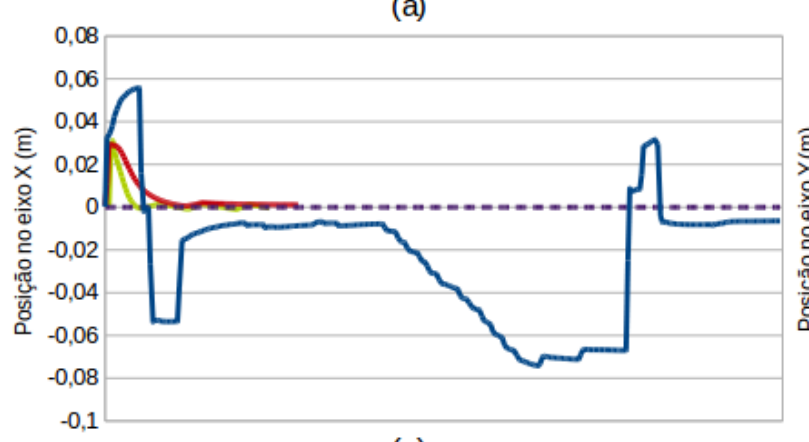

(c)

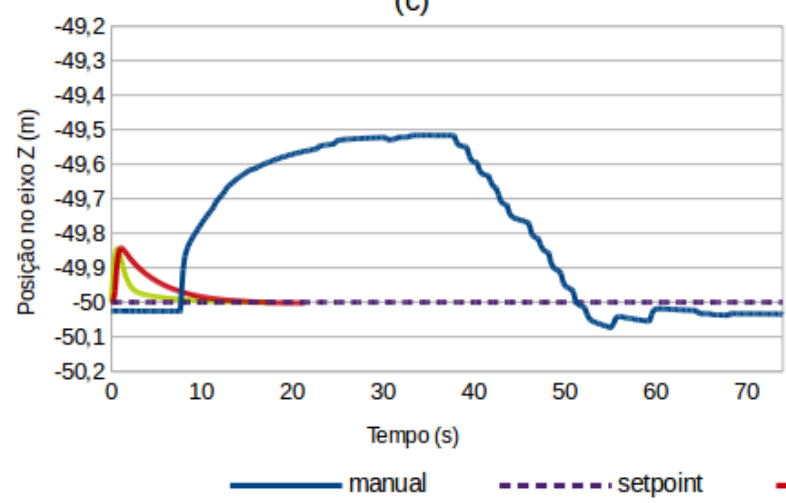

\section{CONCLUSÃO}

Pela análise dos resultados que são demonstrados neste material, é notável que o controlador proporcional apresenta resultados melhores e mais eficientes quando é comparado ao controle manual. A ausência de erro em regime permanente no primeiro teste, também como o desempenho na rejeição da perturbação, comprova a eficiência do controle proporcional. A possibilidade de controlar mais de um grau de liberdade ao mesmo tempo, prova que sua implementação no sistema é viável. Outra vantagem para o controle proporcional é a flexibilidade sobre os valores das velocidades. Enquanto o operador do controle manual fica restrito à sua inferência e percepção humana, o controle proporcional requer baixo processamento computacional, o que dá base para ser facilmente introduzida no ambiente.

\section{REFERÊNCIAS}

${ }^{1}$ A. Sahoo, S. K. Dwivedy, and P. Robi. Advancements in the field of autonomous underwater vehicle. Ocean Engineering, 181:145-160, 2019.

${ }^{2}$ Schjølberg and I. B. Utne. Towards autonomy in rov operations. IFACPapersOnLine, 48(2):183-188, 2015. 
${ }^{3}$ M. M. M. Manhaes, S. A. Scherer, M. Voss, L. R. Douat, and T. Rauschenbach. UUV simulator: A gazebo-based package for underwater intervention and multirobot simulation. In OCEANS 2016 MTS/IEEE Monterey. IEEE, sep 2016.

${ }^{4}$ M. Quigley, K. Conley, B. P. Gerkey, J. Faust, T. Foote, J. Leibs, R. Wheeler, and A. Y. Ng. Ros: an open-source robot operating system. In ICRA Workshop on Open Source Software, 2009.

${ }^{5} \mathrm{~N}$. Koenig and A. Howard. Design and use paradigms for gazebo, an open-source multi-robot simulator. In 2004 IEEE/RSJ International Conference on Intelligent Robots and Systems (IROS) (IEEE Cat. No.04CH37566), volume 3, pages 21492154 vol.3, Sep. 2004.

${ }^{6}$ C. A. Smith and A. B. Corripio. Princípios e práticas do controle automático de processo. LTC, Rio de Janeiro, 3. ed.- edição, 2006. 